\title{
General Anesthesia with Target Controlled Infusion of Propofol and Remifentanil for Planned Caesarean Section in a Parturient with Unruptured Intracranial Aneurysm
}

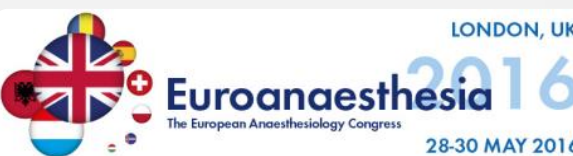

Moreira J. ${ }^{1}$, Pinheiro F. ${ }^{2}$, Amorim P. ${ }^{1}$

${ }^{1}$ Centro Hospitalar do Porto, Dept of Anaesthesiology, Porto, Portugal; ${ }^{2}$ Centro Hospitalar e Universitário de Coimbra, Dept of Anaesthesiology, Coimbra, Portugal

What do we know about unruptured Intracranial Aneurysms?

The incidence of cerebral aneurysm in general population is $2 \%$.

In pregnancy the incidence is similar, but the rupture is more common.

Rupture causes subarachnoid hemorrhage. Regardless of the anesthetic technique, the main objective is to maintain stable the transmural pressure.
Which anesthetic technique

for a planned caesarean section?

General Vs Spinal/epidural

No formal recommendations or guidelines for patients with unruptured intracranial aneurysms.

\section{Case description}

30 Yo $\$, 39$ weeks gestation

Admitted for elective caesarean section.

Indication : unrupted intravavernous carotid aneurysm.

History of left anterior cerebral artery aneurysm clipping 1 year before.

Without any other known pathology.
Premedication:

3omL PO NaCitrate 0.3M

1omg EV Metoclopramide

5omg EV Ranitidine

$+2 \mathrm{~g}$ Cefazolin
Monitoring:

Pulse oximetry

Bispectral índex depth of anestesia Peripheral body temperature,

Continuous direct arterial press

\section{5 channel ECG}

Neuromuscular function Diuresis

TCl Remi Ce 2,5 ng/mL

puncture for arterial line

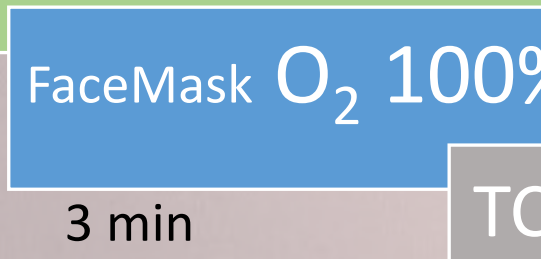

Ce 4

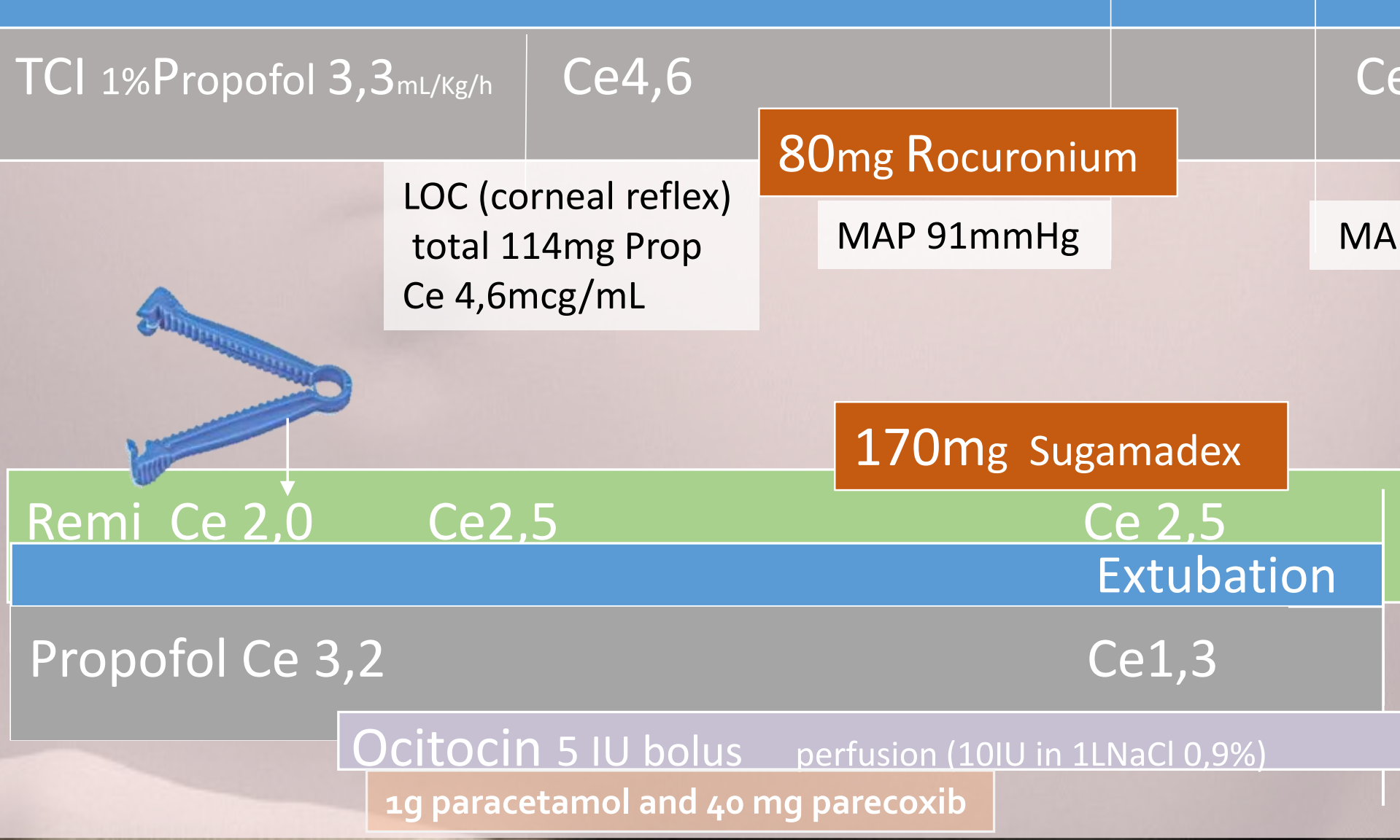

Ce 2,0

$\mathrm{Ce} 3,2$

MAP104mmHg

...9 min after intubation

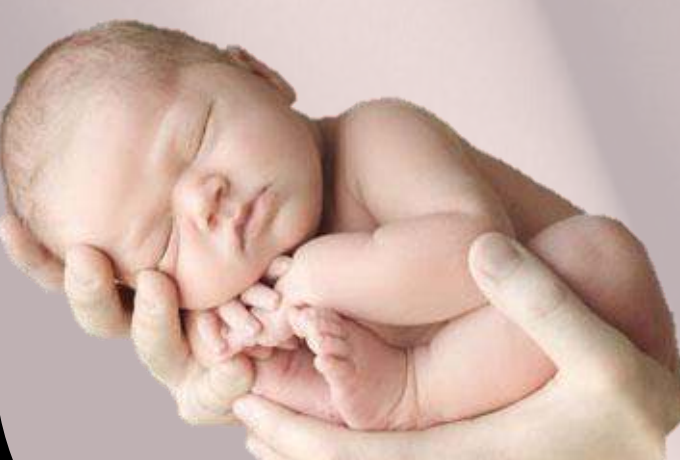
$3395 \mathrm{~g}$ Apgar 5 1min $95 \mathrm{~min}$ Assisted mask ventilation $1 \mathrm{~min} 45 \mathrm{sec}$ No further medical intervention

General Anesthesia in Obstetrics

-Airway management: oropharyngeal edema;

-Increased risk for aspiration;

- Faster desaturation in apnea.

Neuraxial Anesthesia in Cerebral Aneurysm

Substancial leakage of cerebrospinal fluid following dural punction can cause lowering intracranial pressure, thereby increasing wall stress and the risk of aneurysm rupture.
Choice of anesthetic management in this case

-Intubation and skin incision represent noxious stimuli that may result in aneurysm rupture;

- Opioids obtund this response, but may depress the fetus;

-Remi by $\mathrm{TCl}$ allows tight control of concentrations;

-FDA information : Remi Ce of $2 \mathrm{ng} / \mathrm{mL}$ does not cause respiratory depression in the adult. In the fetus Remi elimination is fast.

\section{Learning Points}

In a caesarean section in a parturient with a cerebral aneurysm Total intravenous anaesthesia with $\mathrm{TCl}$ of Remi allowed titration of the opioid concentration in order to obtund sympathetic responses to intubation and incision providing safe maternal anesthesia with minimal side effects in the neonate. 